\title{
SELETIVIDADE FISIOLÓGICA DE AFICIDAS SOBRE JOANINHA CYCLONEDA SANGUINEA (LINNAEUS, 1763) (COLEOPTERA, COCCINELLIDAE) EM ALGODOEIRO
}

\section{J.R. Scarpellini}

Pólo Regional de Desenvolvimento dos Agronegócios do Centro Leste, Av. Bandeirantes, 2419, CEP14001-970, Ribeirão Preto, SP, Brasil. E-mail: jrscarpellini@apta.sp.gov.br

\section{RESUMO}

Com o objetivo de verificar o efeito do tiametoxam, diafentiuron e pimetrozina sobre a joaninha Cycloneda sanguinea e sua presa Aphis gossypii em algodoeiro cultivar Deltaopal foi realizado este estudo, em Ribeirão Preto, SP. Foram testados os seguintes tratamentos (g i.a./ha): Tiametoxan 250 WG (50,0); Pimetrozina 500 PM (100); Diafentiuron 500 SC (250); Tiametoxan 250 WG mais Pimetrozina 500 PM (42,5 + 75); Carbosulfano (160); Imidacloprido 200 SC (50) e testemunha. Foram amostradas 25 plantas inteiras por parcela, avaliando-se o número de pulgões. O estudo de seletividade constou de aplicação sobre as joaninhas e plantas com pulgões; aplicação em plantas com pulgões e colocação das joaninhas nas gaiolas $(15 \times 30 \mathrm{~cm})$, trabalhando com amostras de cinco insetos adultos, além de ensacamento (filós) de 4 plantas por parcela com cinco joaninhas antes da aplicação bem como após a aplicação, no experimento de campo. Avaliou-se a sobrevivência até aos 5 dias após a aplicação ou exposição ao tratamento. No campo, verificouse bom controle do pulgão, não diferindo os tratamentos entre sí, mas todos diferiram da testemunha, enquanto, para joaninha, todos os tratamentos não diferiram entre sí no primeiro dia após a aplicação, tanto sob aplicação direta, quanto exposição indireta. Nos dias subseqüentes ocorreu muito mais mortalidade que no mesmo tipo de experimento em laboratório, quando o tiametoxam, pimetrozina e diafentiuron mostraram menor efeito sobre estes predadores, em relação ao padrão carbosulfano.

PALAVRAS-CHAVE: Joaninhas, algodão, seletividade, Tiametoxam, Pimetrozina, Diafentiuron.

\section{ABSTRACT}

SELECTIVITY OF PESTICIDES ON LADY BEETLES CYCLONEDA SANGUINEA (LINNAEUS, 1763) (COLEOPTERA, COCCINELLIDAE) IN COTTON PLANT. With the objective to verify the effect of Thiamethoxam, Pymetrozine and Diafentiuron on Aphis gossypii and ladybeetles in cotton plant 'Deltaopal' this study was conducted, in Ribeirão Preto, SP, Brazil. The following treatments were tested (g i.a/ha): thiamethoxam 250 WG (500); Pymetrozine 500 PM (100); Diafentiuron 500 SC (250); Thiamethoxam 250 WG more Pymetrozine 500 PM (42,5 + 75); Carbosulfan (160); Imidacloprid 200 SC (50) and untreated. It was examined 25 whole plants per plot, counting the number of aphids present in this. The selectivity study consisted of application on the ladybeetles and plant with aphids; application in plants with aphids and placement of the ladybeetles in the cages $(15 \times 30 \mathrm{~cm}$, working with samples of five adult insects). Four plants per plot were covered with tulles with five ladybeetles inside, before the application as well as after the field application. The survival was evaluated up to 5 days after application or exposure to the treatment. In the field, good control of the aphids was verified, not differing the treatments, but of the check, while for lady beetles, all of the treatments didn't differ among them in the first day after the application, so much under direct application, as indirect exposure. By the subsequent days higher mortality was observed, by the same test made in laboratory, when the thiamethoxam, pymetrozine and diafentiuron showed smaller effect on the predator, than carbosulfan.

KEY WORDS: Lady beetles, cotton, selectivity, Thiamethoxam, Pymetrozine, Diafentiuron.

\section{INTRODUÇÃO}

Dentre as muitas pragas presentes no algodoeiro, no inicio da safra (GALloet al., 2002) sempre ocorre o ataque do pulgão $A$. gossypii Glover, que teve sua importância aumentada com a utilização das variedades do grupo 'deltapine', muito susceptíveis a viroses (DEGRANDE, 1998), sendo a espécie de afídeo mais comum no algodoeiro (HENEBERRY; JECH, 2001). Esses afídeos, além de inocularem vírus, produzem 
uma secreção adocicada, pegajosa e brilhante, o "honeydew", que atrai formigas e contribui para o desenvolvimento de fungos do gênero Capnodium, conhecidos como fumagina (MATTHEWs, 1989;SANTOS, 1999) que prejudicam a respiração e a fotossíntese das folhas. Quando ocorrem na fase final da cultura, depreciam a qualidade da fibra pela produção excessiva de "honeydew" (KABissaet al., 1996), afetando sua utilização industrial (SANTOS, 1999). Para o controle do pulgão-do-algodoeiro, têm sido recomendados sistemas de manejos que utilizam o controle cultural, cultivares resistentes e o biológico WeathersbeE; Hardee, 1994; Vergara; Galeano, 1994; Isikber; Copland, 2002).

A abundância eimportância dosinsetos predadores variam consideravelmente de ano para ano e de local para local, de acordo com GRAVENA; STERLING (1983). Pesquisas sobreos efeitoscolaterais deagroquímicosem organismos benéficos têm se tornado obrigatórias em diversos países, fazendocom queseestabeleçamlinhas de ação internacionalmente aprovadas e em regime de urgência, oferecendoaosusuários dessesinsumosmais informações para emprego em programas de manejo de pragas (HAssANetal., 1994). No Brasil não existe padronização de procedimentos de pesquisa para avaliar seletividade sobre a maioria dos organismos benéficos nos diferentes cultivos (Maisetal., 2001). Alémdisso,não se leva muito em consideração o efeito colateral dos defensivos nos artrópodes predadores presentes no algodoeiro, o que pode causar surtos de pragas secundarias, ressurgência e resistência das pragas (CAMPOs et al., 1986).

GRAVENA (1990) enumerou vários predadorescomo mais importantes e abundantes na cultura algodoeira, especialmenteCycloneda sanguinea. De acordocom OBRYCHI; KRING (1998), as joaninhas são reconhecidas como importantes inimigos naturais de pragas, como pulgões e cochonilhas. Citaram que estes predadores apresentam excelente capacidade de dispersão no campo e podem ser favorecidas com a criação de ambientes para refúgio, oferecendo condições de reprodução. A manutenção e o incremento da população destes inimigos naturais nas lavouras são obtidos com a utilização de variedades que oferecem melhor resistência às pragas, contribuindo para a redução de aplicação de inseticidas. No controle biológico de $A$. gossypii, as joaninhas são um de seus inimigos naturais (Penna, 1998), principalmente as espécies Cycloneda sanguinea (Linnaeus) e Hippodamia convergens Guérin-Meneville SOAREs; BusOLI, 1995; Guerreiro et al., 2002). De acordo comGravena (1983), a maior abundância de predadores coincide com a ocorrência de pulgões, ou seja, dos 30 aos 80 dias após o plantio, embora alguns autores denotam um pico dos 80-90 dias após o plantio, em função do ataque de lagartas. A abundância de insetos predadores será menor se a população de presas herbívoras fosse reduzida (Gould et al., 1991). Apesar de predadores polífagos e generalistas representarem um importante fator de mortalidade da praga (RAMALHo et al. 1990), não são capazes de regular populações de A. gossypii. (SujII et al., 2005). BoIçA Junior et al. (2004) observaram, em condições de casa de vegetação, que adultos de $C$. sanguinea reduziram em $93,5 \%$ a população de pulgões $A$. gossypii, em apenas dois dias.

SOARES; BUSOLI (2000) verificaram que fipronil provoca impacto menor em população de C. sanguinea do que endosulfan, logo após a aplicação (até 24h). SCARPELLINI et al. (2001) verificaram que a recuperação do complexo de predadores, após a aplicação do carbosulfano 400 SC a 120 gi.a./ ha e do acetamiprido a $20 \mathrm{~g}$ i.a./ha, entre outros, normaliza-se depois dos sete dias da aplicação. SCARPELLINI et al. (2005) observaram a ação de flonicamida, concluindo que, embora colabore para redução da joaninha predadora $C$. sanguinea, importante no controle natural do pulgão, tem maior seletividade que os padrões (carbosulfano e imidacloprido) utilizados, facilitando a recuperação populacional desses organismos mais rapidamente.

O presente trabalho teve por objetivo estudar o efeito do thiamethoxam, diafentiuron e pymetrozine sobre joaninhas Cycloneda sanguinea L. (Coleoptera, Coccinellidae) e sua presa Aphis gossypii em algodoeiro cultivar Deltaopal.

\section{MATERIAL E MÉTODOS}

Os experimentos realizados (um de campo, um semicampo e dois em casa de vegetação e laboratório) foram em blocos ao acaso, constando de sete tratamentos e quatro repetições, sendo utilizado o cultivar Deltaopal, não sendo feito tratamento de sementes e tratos culturais de acordo com recomendações (BoLSA DE MERCADORIAS \& FUTURO, 1992), sendo as parcelas de 10 linhas por $10 \mathrm{~m}\left(80 \mathrm{~m}^{2}\right)$, onde foram testados os tratamentos que constam da Tabela 1 .

\section{Experimento de campo}

As pulverizações foram realizadas utilizando-se um pulverizador costal $\mathrm{CO} 2$, a pressão constante de $40 \mathrm{lb} / \mathrm{pol}^{2}$ e bico TLX3, com volume de calda de 200 L/ha, anotando-se o horário de aplicação, umidade relativa, temperatura e velocidade do vento. Foi utilizado algodoeiro Deltaopal, com 30 dias após a germinação, que já apresentava infestação de pulgão, e parcelas de $10 \times 10 \mathrm{~m}$. Foram colocados, antes da aplicação (realizada em 10/1/2005), 6 saquinhos de filó $(15 \times 30 \mathrm{~cm})$ contendo 5 joaninhas em cada. Uma hora após a aplicação foram colocados mais 4 saquinhos de filó (marcados com cor diferente) por parcela. 
Tabela 1 - Tratamentos e dosagens usados no estudo. Ribeirão Preto, SP, 2005.

\begin{tabular}{|c|c|c|c|c|}
\hline \multirow[b]{2}{*}{$\mathrm{N}^{\mathrm{o}}$} & \multicolumn{2}{|c|}{ Tratamentos } & \multicolumn{2}{|c|}{ Dosagens } \\
\hline & Produtos & Ingrediente ativo & g i.a./ha & g p.c./ha \\
\hline 1 & Actara $250 \mathrm{WG}$ & Tiametoxam & 50,0 & 200 \\
\hline 2 & Chess 500 PM & Pimetrozina & 100 & 200 \\
\hline 3 & Polo 500 SC & Diafentiurom & 250 & 500 \\
\hline 4 & Actara $250 \mathrm{WG}+$ Chess $500 \mathrm{PM}$ & Tiametoxam + Pimetrozina & $42,5+75$ & $170+150$ \\
\hline 5 & Marshal 400 SC & Carbosulfano & 160 & 400 \\
\hline 6 & Provado 200 SC & Imidacloprido & 50 & 250 \\
\hline 7 & Testemunha & -------- & ---- & ---- \\
\hline
\end{tabular}

As amostragens para o pulgão foram feitas em 25 plantas inteiras por parcela (experimentos realizados de 10 a 18/1/2005) e, no caso da avaliação da seletividade fisiológica da joaninha $C$. sanguinea, foi feito no campo observação do efeito tópico (observação da sobrevivência de joaninhas em 4 saquinhos de filó, por parcela, colocados antes da aplicação). Procurou-se avaliar a sobrevivência até 5 dias após a aplicação ou exposição ao tratamento.

Observou-se também o efeito da exposição indireta, por contato e ingestão, avaliando-se a sobrevivência das joaninhas nos saquinhos de filó, colocadas após a aplicação, em saquinhos com cor diferente.

\section{Experimento semicampo}

Realizou-se um experimento de semicampo, representado por dois dos saquinhos com joaninhas que teve exposição direta e foram levados ao laboratório (depois de uma hora), colocados em condições ideais, com plantas de algodão contendo pulgões (vasos não pulverizados) em gaiolas de $20 \times 30 \mathrm{~cm}$, sem contaminação alguma com defensivo e oferecidas presas não contaminadas, avaliando-se a sobrevivência das joaninhas por cinco dias.

\section{Experimentos de laboratório e casa de vegetação}

a) Exposição direta: aplicação de vasos contendo plantas de algodão com 15 dias após a germinação e pulgões, junto com 5 joaninhas [acondicionadas por um filó de voil $(15 \times 30 \mathrm{~cm})]$, utilizando-se um pulverizador costal CO2, a pressão constante de $40 \mathrm{lb} / \mathrm{pol}^{2}$ e bico TLX3, com volume de calda de $200 \mathrm{~L} /$ ha, simulando as condições do campo. Após a aplicação, as plantas e joaninhas eram acondicionadas em gaiolas $(20 \times 30 \mathrm{~cm})$, observando-se a sobrevivência aos 1, 2, 3 e 5 dias após. Se necessário, eram oferecidas presas suplementares às joaninhas.

b) Exposição indireta: aplicação de vasos contendo plantas de algodão com 15 dias após a germinação e pulgões, utilizando-se um pulverizador costal CO2, a pressão constante de $40 \mathrm{lb} /$ pol$^{2}$ e bico TLX3, com volume de calda de $200 \mathrm{~L} / \mathrm{ha}$, simulando as condições do campo, sendo depois acondicionadas em gaiolas $(20 \times 30 \mathrm{~cm})$, observando-se a sobrevivência aos 1,2,3 e 5 dias após. Se necessário, eram oferecidas presas suplementares às joaninhas.

Para evitar o condicionamento pré-imaginal (SOARES; BusOLI, 2000)coletoram-sejoaninhas, de uma área reserva (pomar de 2 ha), contendo losna branca Parthenium hysterophorus L., atacada pelo pulgão Uroleucon ambrosiae, que serviu de presa para criação da joaninha C. sanguinea (presa ecultura diferentes da estudada).

\section{RESULTADOS E DISCUSSÃO}

Todos os tratamentos apresentaram eficiência de controle satisfatória do pulgão-das-inflorescências Aphis gossypii até os 12 dias após a aplicação (Tabela 2), embora o melhor efeito desses produtos tenha sido aos 4 e 7 dias, proporcionando todos mais de $90 \%$ de eficiência, ideal para um inseto vetor. $\mathrm{Na}$ avaliação realizada 1 dia após a aplicação, todos os tratamentos diferiram da testemunha, mas não entre sí, embora a eficiência satisfatória para um inseto vetor seja de $90 \%$, valor apresentado apenas pelo Carbosulfano 400SCa $160 \mathrm{~g}$ i.a./ha, denotando os demais uma ação menos rápida, dado serem produtos queagem na fisiologia do inseto, conseqüentemente com menor efeito de choque. Resultados semelhantes foram obtidos porSCARPELLINI et al. (2005), observando menor efeito de choque utilizando juvenóides do que Carbosulfano, mas obtendo bom controle do pulgão com ambos.

Pelas Tabelas 3 e 4, observa-se que a seletividade desses produtos para a joaninhaC. sanguinea, nocampo, é menor do que aquela apresentada em laboratório (Tabelas5e6), tendoasjoaninhas sido bastanteafetadas pelaaplicaçãodireta, commaior efeito para Carbosulfano 400 SCa 160 gi.a./ ha e Imidacloprido 200SCa 50 gi.a./ ha, que diferiram significativamente da testemunha. Tiametoxam 250 WGa 50 gi.a.ha, Pimetrozina 500 PM 
a 100 g i.a./ha e Diafentiuron 500 SC a 250 g i.a./ha não diferiram significativamente da testemunha, na aplicação direta no campo, considerando que os predadores resistiramaté3 diasapósaaplicação, quandodadaafalta dealimento(Gould etal.,1991) easintempéries dotempo, tenderama perecer,mascomefeitomenornostratamentos citados que nos demais. Resultados semelhantes foram obtidos quando da exposição indireta, quando apenas Carbosulfano400SCa160 gi.a./ha eImidacloprido 200 $\mathrm{SC}$ a $50 \mathrm{~g}$ i.a./ha diferiram significativamente da testemunha, ate aos 5 dias, com menor efeito do Tiametoxam 250 WG 50 gi.a.ha, Pimetrozina 500 PMa 100 gi.a./ha e Diafentiuron 500 SC a $250 \mathrm{~g}$ i.a./ha.

ScARPELlini etal.(2003), em avaliação da ocorrência no campo, verificaram que para o complexo de joaninhas, o carbosulfano a 120 gi.a./ha; o tiametoxam a $30 \mathrm{~g}$ i.a./ha e o acetamiprido a $30 \mathrm{~g}$ i.a./ha não apresentaram diferenças significativas entre si e, embora reduzissem a população destes predadores, a partir dos 7 dias após a aplicação, observou o restabelecimento da população no mesmo nível da testemunha. Esta diferença entre laboratório e campo écorroborada pelo experimento cujo resultadoéapresentado na Figura 1, onde os predadores adultos foram pulverizados no campo e transferidos para condições ideais no laboratório, com muito menor efeito dos agroquímicos, que aqueles onde os insetos continuaram nas plantas pulverizadas, embora, Carbosulfano400SCa 160 gi.a./ha também apresentou maior efeito que os demais tratamentos, menos drástico que a situação anterior, simulando melhor o que acontece no campo, pois em condições adversas, estes predadores generalistas vão procurar outros hospedeiros. Por outro lado, quanto melhor o controle dos pulgões, menor a presença des ses predadores nas parcelas tratadas, haja vista não ter alimento disponível (pulgões) conformeGould et al. (1991), que citaram que a abundância de insetos predadores será menor à medida que haja redução de presas herbívoras.

Nas avaliações em gaiola, em condições de laboratório (Tabelas 5 e 6), não se observou efeito significativo sobre a mortalidade de C. sanguinea, em exposição indireta (joaninhas sobre plantas de algodão, com pulgões aplicados), até os dois dias após a aplicação, excetuando-se o tratamento Carbosulfano 400 SCa 160 gi.a./ha, que apresentou maior mortalidade e diferiu da testemunha. Aos 3 e 5 dias após a aplicação, os tratamentos diferiram da testemunha, embora com sobrevivência de adultos muito altas, em relação ao mesmo teste realizado no campo.

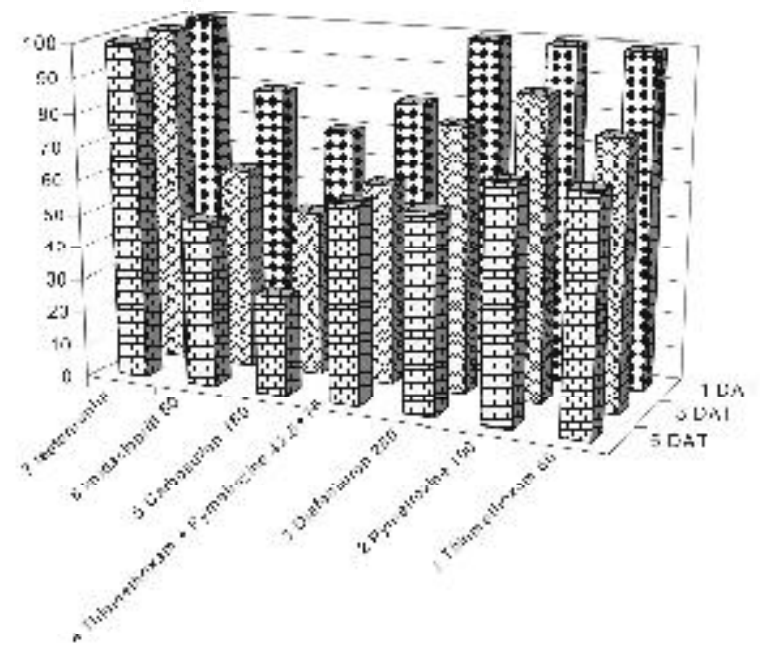

Fig. 1 - Porcentagem de sobrevivência de joaninhas $C$. sanguinea (adultos), por tratamento, nas avaliações realizadas aos 1, 3 e 5 dias após o tratamento (DAT). Experimento semi campo, de exposição direta (joaninhas em grupos de 5 sob aplicação no campo e transferidas para gaiolas de 15 × 30 cm). Ribeirão Preto, SP, 10 a 16/1/2005.

Tabela 2 - Número de pulgões $A$. gossypiiencontrados por tratamento (experimento de campo) nas avaliações realizadas aos 1, 4, 7 e 12 dias após o tratamento (DAT). Ribeirão Preto, SP, 10 a 22/1/2005.

\begin{tabular}{|c|c|c|c|c|c|c|c|c|c|c|}
\hline \multirow{3}{*}{$\mathrm{N}^{\mathrm{o}}$} & \multirow{3}{*}{$\begin{array}{l}\text { Tratamentos } \\
\text { Produtos } \\
\text { Tiametoxam 250 WG }\end{array}$} & \multicolumn{9}{|c|}{ Avaliações } \\
\hline & & \multirow{2}{*}{$\frac{\text { g i. a./ha }}{50,0}$} & \multicolumn{2}{|c|}{$1 \mathrm{DAT}$} & \multicolumn{2}{|c|}{$4 \mathrm{DAT}$} & \multicolumn{2}{|c|}{$7 \mathrm{DAT}$} & \multicolumn{2}{|c|}{$12 \mathrm{DAT}$} \\
\hline & & & $175^{1} \mathrm{~b}$ & $81^{2}$ & $23 \mathrm{~b}$ & 98 & $31 \mathrm{~b}$ & 97 & $78 \mathrm{~b}$ & 89 \\
\hline 2 & Pimetrozina 500 PM & 100 & $191 \mathrm{~b}$ & 79 & $51 \mathrm{~b}$ & 95 & $29 \mathrm{~b}$ & 97 & $73 \mathrm{~b}$ & 90 \\
\hline 3 & Diafentiuron 500 SC & 250 & $189 \mathrm{~b}$ & 80 & $45 \mathrm{~b}$ & 96 & $41 \mathrm{~b}$ & 95 & $82 \mathrm{~b}$ & 89 \\
\hline 4 & Tiametoxam 250 WG + Pimetrozina 500 PM & $42,5+75$ & $109 \mathrm{~b}$ & 88 & $34 \mathrm{~b}$ & 97 & $25 \mathrm{~b}$ & 97 & $50 \mathrm{~b}$ & 93 \\
\hline 5 & Carbosulfano $400 \mathrm{SC}$ & 160 & $88 \mathrm{~b}$ & 91 & $10 \mathrm{~b}$ & 99 & $26 \mathrm{~b}$ & 97 & $58 \mathrm{~b}$ & 92 \\
\hline 6 & Imidacloprido 200 SC & 50 & $156 \mathrm{~b}$ & 83 & $58 \mathrm{~b}$ & 95 & $42 \mathrm{~b}$ & 95 & $70 \mathrm{~b}$ & 90 \\
\hline 7 & Testemunha & - & 930 a & - & $1100 \mathrm{a}$ & - & $900 \mathrm{a}$ & - & $720 \mathrm{a}$ & - \\
\hline \multicolumn{2}{|r|}{ Coeficiente de variação (\%) } & 20,64 & \multicolumn{2}{|c|}{27,76} & \multicolumn{2}{|l|}{21,49} & \multicolumn{2}{|c|}{18,53} & & \\
\hline \multicolumn{2}{|c|}{ F Tratamentos } & $16,57^{* *}$ & \multicolumn{2}{|c|}{$20,77^{* *}$} & \multicolumn{2}{|c|}{$29,37^{* *}$} & \multicolumn{2}{|c|}{$17,97^{* *}$} & & \\
\hline
\end{tabular}

${ }^{1}$ Valores na mesma coluna, seguidas de letras iguais, não diferem entre sí por Tukey (5\%).

${ }^{2}$ Porcentagem de redução populacional, em relação à testemunha. 
Tabela 3 - Número de joaninhas C. sanguinea (adultos) vivas por tratamento nas avaliações realizadas aos 1, 3 e 5 dias após o tratamento (DAT). Ensaio de campo, aplicação direta (joaninhas em saquinhos de filó, colocadas antes da aplicação). Ribeirão Preto, SP, 10 a 16/1/2005.

\begin{tabular}{|c|c|c|c|c|c|c|c|c|}
\hline \multirow{3}{*}{$\frac{\mathrm{N}^{\mathrm{o}}}{1}$} & \multirow{3}{*}{$\begin{array}{ll} & \text { Tratamentos } \\
\text { Produtos } & \\
\text { Tiametoxam 250 WG }\end{array}$} & \multicolumn{7}{|c|}{ Avaliações } \\
\hline & & \multirow{2}{*}{$\frac{\text { g i. a./ha }}{50,0}$} & \multicolumn{2}{|c|}{$1 \mathrm{DAT}$} & \multicolumn{2}{|c|}{3 DAT } & \multicolumn{2}{|c|}{$5 \mathrm{DAT}$} \\
\hline & & & $10^{1} \mathrm{~b}$ & $44^{2}$ & $8^{1} \mathrm{ab}$ & $47^{2}$ & $6^{1} \mathrm{ab}$ & $33^{2}$ \\
\hline 2 & Pimetrozina 500 PM & 100 & $10 \mathrm{~b}$ & 44 & $9 \mathrm{ab}$ & 40 & $6 \mathrm{ab}$ & 33 \\
\hline 3 & Diafentiuron 500 SC & 250 & $9 \mathrm{~b}$ & 50 & $8 \mathrm{ab}$ & 47 & $5 \mathrm{ab}$ & 44 \\
\hline 4 & Tiametoxam 250 WG + Pimetrozina 500 PM & $42,5+75$ & $9 \mathrm{~b}$ & 50 & $7 \mathrm{~b}$ & 53 & $5 \mathrm{ab}$ & 44 \\
\hline 5 & Carbosulfano $400 \mathrm{SC}$ & 160 & $10 \mathrm{~b}$ & 44 & $5 \mathrm{~b}$ & 67 & $4 \mathrm{~b}$ & 56 \\
\hline 6 & Imidacloprido 200 SC & 50 & $9 \mathrm{~b}$ & 50 & $5 \mathrm{~b}$ & 67 & $4 \mathrm{~b}$ & 56 \\
\hline 7 & Testemunha & - & 18 a & - & $15 \mathrm{a}$ & - & $9 \mathrm{a}$ & - \\
\hline \multicolumn{2}{|c|}{ Coeficiente de variação (\%) } & 10,09 & \multicolumn{2}{|c|}{15,63} & \multicolumn{2}{|c|}{11,30} & & \\
\hline \multicolumn{2}{|c|}{ F Tratamentos } & $5,57^{* *}$ & \multicolumn{2}{|c|}{$4,71^{* *}$} & \multicolumn{2}{|c|}{$3,12^{*}$} & & \\
\hline
\end{tabular}

${ }^{1}$ Valores na mesma coluna, seguidas de letras iguais, não diferem entre sí por Tukey (5\%).

${ }^{2}$ Porcentagem de redução populacional, em relação à testemunha.

Tabela 4 - Número de joaninhas C. sanguinea (adultos) vivas no tratamento nas avaliações realizadas aos 1,3 e 5 dias após o tratamento (DAT) (joaninhas em saquinhos filó, colocadas após a aplicação). Ribeirão Preto, SP, 10 a 16/1/005.

\begin{tabular}{|c|c|c|c|c|c|c|c|c|}
\hline \multirow{3}{*}{$\frac{\mathrm{N}^{\mathrm{o}}}{1}$} & \multirow{3}{*}{$\begin{array}{l}\text { Tratamentos } \\
\text { Produtos } \\
\text { Tiametoxam 250 WG }\end{array}$} & \multicolumn{7}{|c|}{ Avaliações } \\
\hline & & \multirow{2}{*}{$\frac{\text { g i. a./ha }}{50,0}$} & \multicolumn{2}{|c|}{$1 \mathrm{DAT}$} & \multicolumn{2}{|c|}{$3 \mathrm{DAT}$} & \multicolumn{2}{|c|}{$5 \mathrm{DAT}$} \\
\hline & & & $19^{1} \mathrm{ab}$ & $5^{2}$ & $17^{1} \mathrm{a}$ & $11^{2}$ & $12^{1} \mathrm{ab}$ & $29^{2}$ \\
\hline 2 & Pimetrozina 500 PM & 100 & 20 a & 0 & $16 \mathrm{ab}$ & 16 & $13 \mathrm{ab}$ & 24 \\
\hline 3 & Diafentiuron 500 SC & 250 & $19 \mathrm{ab}$ & 5 & $16 \mathrm{ab}$ & 16 & $12 \mathrm{ab}$ & 29 \\
\hline 4 & Tiametoxam 250 WG + Pimetrozina 500 PM & $42,5+75$ & $20 \mathrm{a}$ & 0 & $15 \mathrm{~b}$ & 21 & $13 \mathrm{ab}$ & 29 \\
\hline 5 & Carbosulfano 400 SC & 160 & $17 \mathrm{~b}$ & 15 & $6 \mathrm{c}$ & 68 & $3 \mathrm{c}$ & 82 \\
\hline 6 & Imidacloprido 200 SC & 50 & $19 \mathrm{ab}$ & 5 & $12 \mathrm{bc}$ & 37 & $9 \mathrm{~b}$ & 47 \\
\hline 7 & Testemunha & - & $20 \mathrm{a}$ & - & $19 \mathrm{a}$ & -- & $17 \mathrm{a}$ & - \\
\hline \multirow{2}{*}{\multicolumn{2}{|c|}{$\begin{array}{l}\text { Coeficiente de variação (\%) } \\
\text { F Tratamentos }\end{array}$}} & 13,01 & \multicolumn{2}{|c|}{24,62} & \multicolumn{2}{|c|}{10,68} & & \\
\hline & & $3,00^{*}$ & \multicolumn{2}{|c|}{$42,26 * *$} & \multicolumn{2}{|c|}{$13,54^{* *}$} & & \\
\hline
\end{tabular}

${ }^{1}$ Valores na mesma coluna, seguidas de letras iguais, não diferem entre sí por Tukey (5\%).

${ }^{2}$ Porcentagem de redução populacional, em relação à testemunha.

Tabela 5 - Número de joaninhas C. sanguinea (adultos) vivas por tratamento nas avaliações realizadas aos 1, 2, 3 e 5 dias após o tratamento (DAT). Ensaio de laboratório de exposição indireta (joaninhas em grupos de 5 em gaiolas de $15 \times 30$ cm). Ribeirão Preto, SP, 6/1 a 11/1/2005.

\begin{tabular}{|c|c|c|c|c|c|c|c|c|c|}
\hline \multirow{3}{*}{$\begin{array}{ll} & \text { Tratamentos } \\
1 & \text { Tiametoxam 250 WG }\end{array}$} & \multicolumn{9}{|c|}{ Avaliações } \\
\hline & \multirow{2}{*}{$\frac{\text { g i. a./ha }}{50,0}$} & \multicolumn{2}{|c|}{$1 \mathrm{DAT}$} & \multicolumn{2}{|c|}{$2 \mathrm{DAT}$} & \multicolumn{2}{|c|}{$3 \mathrm{DAT}$} & \multicolumn{2}{|c|}{$5 \mathrm{DAT}$} \\
\hline & & $17^{1} \mathrm{a}$ & $15^{2}$ & $13^{1} \mathrm{ab}$ & $28^{2}$ & $12^{1} \mathrm{~b}$ & $33^{2}$ & $10^{1} \mathrm{~b}$ & $33^{2}$ \\
\hline 2 Pimetrozina 500 PM & 100 & $15 \mathrm{ab}$ & 25 & $15 \mathrm{a}$ & 17 & $13 \mathrm{~b}$ & 28 & $9 \mathrm{~b}$ & 40 \\
\hline 3 Diafentiuron 500 SC & 250 & $12 \mathrm{~b}$ & 40 & $14 \mathrm{ab}$ & 22 & $12 \mathrm{~b}$ & 33 & $8 \mathrm{~b}$ & 47 \\
\hline 4 Tiametoxam 250 WG + Pimetrozina 500 PM & $42,5+75$ & $12 \mathrm{~b}$ & 40 & $12 \mathrm{ab}$ & 33 & $11 \mathrm{~b}$ & 39 & $7 \mathrm{~b}$ & 53 \\
\hline 5 Carbosulfano 400 SC & 160 & $10 \mathrm{c}$ & 50 & $9 \mathrm{~b}$ & 50 & $8 \mathrm{c}$ & 56 & $4 \mathrm{~b}$ & 73 \\
\hline 6 Imidacloprido 200 SC & 50 & 17 a & 15 & $14 \mathrm{ab}$ & 22 & $12 \mathrm{~b}$ & 33 & $8 \mathrm{~b}$ & 47 \\
\hline 7 Testemunha & - & 20 a & - & 18 a & - & 18 a & - & 15 a & - \\
\hline \multicolumn{2}{|l|}{ Coeficiente de variação (\%) } & \multicolumn{2}{|c|}{5,71} & \multicolumn{2}{|l|}{8,68} & \multicolumn{2}{|c|}{4,92} & \multicolumn{2}{|c|}{11,141} \\
\hline F Tratamentos & & \multicolumn{2}{|c|}{$9,22 * *$} & \multicolumn{2}{|c|}{$4,32^{* *}$} & \multicolumn{2}{|c|}{$17,44^{* *}$} & \multicolumn{2}{|c|}{$7,70^{* *}$} \\
\hline
\end{tabular}

${ }^{1}$ Valores na mesma coluna, seguidas de letras iguais, não diferem entre sí por Tukey (5\%).

${ }^{2}$ Porcentagem de redução populacional, em relação à testemunha. 
Tabela 6 - Número de joaninhas C. sanguinea (adultos) vivas por tratamento nas avaliações realizadas aos 1, 2, 3 e 5 dias após o tratamento (DAT). Experimento de laboratório de aplicação direta. Ribeirão Preto, SP, 6/1 a 11/01/2005.

\begin{tabular}{|c|c|c|c|c|c|c|c|c|c|c|}
\hline \multirow{3}{*}{$\frac{\mathrm{N}^{\mathrm{o}}}{1}$} & \multirow{3}{*}{$\begin{array}{l}\text { Produtos } \\
\text { Tiametoxam } 250 \text { WG }\end{array}$} & \multicolumn{9}{|c|}{ Avaliações } \\
\hline & & \multirow{2}{*}{$\frac{\text { g i. a./ha }}{50,0}$} & \multicolumn{2}{|c|}{$1 \mathrm{DAT}$} & \multicolumn{2}{|c|}{$2 \mathrm{DAT}$} & \multicolumn{2}{|c|}{$3 \mathrm{DAT}$} & \multicolumn{2}{|c|}{$5 \mathrm{DAT}$} \\
\hline & & & $16^{1} \mathrm{ab}$ & $16^{2}$ & $14 \mathrm{ab}$ & 26 & $10 \mathrm{ab}$ & 33 & $9 \mathrm{ab}$ & 60 \\
\hline 2 & Pimetrozina 500 PM & 100 & $15 \mathrm{ab}$ & 21 & $11 \mathrm{ab}$ & 42 & $8 a b$ & 47 & $6 \mathrm{~b}$ & 40 \\
\hline 3 & Diafentiuron 500 SC & 250 & $12 \mathrm{~b}$ & 37 & $8 \mathrm{~b}$ & 58 & $6 \mathrm{~b}$ & 60 & $5 \mathrm{~b}$ & 67 \\
\hline 4 & Tiametoxam 250 WG + Pimetrozina 500 PM & $42,5+75$ & $6 \mathrm{c}$ & 68 & $3 c$ & 84 & $2 c$ & 87 & $2 c$ & 87 \\
\hline 5 & Carbosulfano 400 SC & 160 & $5 \mathrm{c}$ & 74 & $3 c$ & 84 & $2 \mathrm{c}$ & 87 & $1 \mathrm{c}$ & 93 \\
\hline 6 & Imidacloprido 200 SC & 50 & $3 c$ & 84 & $2 c$ & 89 & $2 c$ & 87 & $1 \mathrm{c}$ & 93 \\
\hline 7 & Testemunha & - & 19 a & - & $18 \mathrm{a}$ & - & 15 a & - & 15 a & - \\
\hline \multicolumn{2}{|c|}{ Coeficiente de variação (\%) } & & \multicolumn{2}{|c|}{8,68} & \multicolumn{2}{|c|}{14,95} & \multicolumn{2}{|c|}{17,10} & \multicolumn{2}{|c|}{17,22} \\
\hline \multicolumn{2}{|c|}{ F Tratamentos } & & \multicolumn{2}{|c|}{$37,49^{* *}$} & \multicolumn{2}{|c|}{$18,73^{* *}$} & \multicolumn{2}{|c|}{$13,49 * *$} & \multicolumn{2}{|c|}{$16,58^{* *}$} \\
\hline
\end{tabular}

${ }^{1}$ Valores na mesma coluna, seguidas de letras iguais, não diferem entre sí por Tukey (5\%).

${ }^{2}$ Porcentagem de redução populacional, em relação à testemunha.

Resultados semelhantes, mas um pouco mais drásticos, foram obtidos na aplicação direta, em laboratório quando Carbosulfano 400 SC a 160 g i.a./ha, Imidacloprido 200 SC a $50 \mathrm{~g}$ i.a./ ha e tiametoxam 250 WG + Pimetrozina 500 PM a 42,5 + 75 g i.a./ha, apresentaram maior mortalidade, em relação ao demais tratamentos.

SOARES; BusOli (2000) não observaram nenhuma diferença significativa na população da joaninha $C$. sanguinea, em experimentos no campo, com fipronil e endossulfan, utilizando o método do pano de batida como amostragem, a partir de sete dias da aplicação, com maior redução 1 e 3 dias após a aplicação. SujII $e t$ al. (2005) afirmaram que a elevada capacidade de dispersão e redistribuição entre áreas pelos predadores e a baixa densidade da presa afetando a colonização por predadores em processos dependentes podem minimizar as diferenças entre comunidades observadas (com controle químico, biológico e sem controle).

\section{CONCLUSÕES}

Tiametoxam, pimetrozina e diafentiuron, embora colaborem para redução da joaninha predadora $C$. sanguinea, importante no controle natural do pulgão A. gossypii, apresentam seletividade maior que os demais defensivos utilizados, facilitando a recuperação populacional desses organismos mais rapidamente.

Os aficidas tiametoxam, pimetrozina, diafentiuron, carbosulfano e imidacloprido apresentaram bom controle pulgão A. gossypii Glover, 1878, em algodoeiro.

\section{AGRADECIMENTOS}

O autor agradece ao Dr. Luis Cláudio Paterno Silveira (UFLA - Lavras) pela identificação dos pulgões e a Giuliano Roberto Scarpellini pela manutenção das joaninhas no laboratório.

\section{REFERÊNCIAS}

BOIÇA JUNIOR., A.L.; SANTOS, T.M.; KURANISHI, A.K. Desenvolvimento larval e capacidade predatória de Cycloneda sanguinea (L.) e Hippodamia convergens Guerin-Men. Alimentadas com Aphis gossypii Glover sobre cultivares de algodoeiro. Acta Scientiarum Agronomy, v.26, n.2, p.239-244, 2004.

BOLSA DE MERCADORIAS \& FUTUROS (São Paulo, SP). Manual do produtor de algodão. São Paulo, 1992. $158 \mathrm{p}$.

CAMPOS, A.R.; GRAVENA, S.; BERTOZO, R.; BARBIERI, J. Artrópodes predadores na cultura algodoeira e comparação de métodos de amostragem. Anais da Sociedade Entomológica do Brasil, v.15, p.5-20, 1986.

DEGRANDE, P.E. Guia prático de controle das pragas do algodoeiro. Dourados: UFMS/Fundação Chapadão, 1998. $60 \mathrm{p}$.

GALLO, D.; NAKANO, O.; CARVALHO, R.P.L.; DE BAPTISTA, G.C. BERTI FILHO, E.; PARRA, J.R.P.; ZUCCHI, R.A.; ALVES, S.B.; VENDRAMIN, J.D.; MARCHINI, L.C; LOPES, J.R.S.; OMOTO, C. Entomologia agrícola. Piracicaba: Fealq, 2002. 920p. 
GOULD, F.; KENNEDY, G.G.; JOHNSON, M.T. Effects of natural enemies on the rate of herbivore adaptation to resistant host plants. Entomologia Experimentalis et Applicata, v.58, p.1-14, 1991.

GRAVENA, S. O Controle biológico na cultura algodoeira. Informe Agropecuário, v.9, p.3-15, 1983.

GRAVENA, S.; STERLING, W.L. Natural predation on the cotton leaf worm (Lepidoptera: Noctuidae) Journal of Economic Entomology, v.76, p.779-784, 1983.

GRAVENA, S. Estratégias e táticas de MIP algodoeiro no Brasil. In: FERNANDES, O.P.; CORREIA C.B.; BORTOLI, S.A. (Ed.). Manejo integrado de pragas e nematóides. Jaboticabal: Funep, 1990. p.1-14.

GUERREIRO, J.C.; SILVA, R.A.; BUSOLI, A.C.; BERTI FILHO, E. Coccinelídeos predadores que ocorrem no estágio inicial da cultura do algodoeiro em Jaboticabal, SP, Brasil. Revista de Agricultura, v. 77, n.1, p.161-168, 2002.

HASSAN, S.A.; BIGLER, F.; BOGENSCHUTZ, H.; BOLLER, E.; BRUN, J.; CALIS, J.N.M.; COREMANSPELSENEER, J.; DUSO, C.; GROVE, A.; HEINBACH, U.J.; HELYER, N.; HOKKAMEN, H.; LEWIS, G.B.; MANSOUR, F.; MORETH, L.; POLGAR, L.; SANSOEPETERSEN, L.; SAUPHANOR, B.; STAUBLI, A.; STERK, G.; VAINIO, A.; VAN DE VEIRE, M.; VIGGIANI G.; VOGHT, H. Results of the sixth joint pesticide testing programme of the IOBC/WPRS Working Group "Pesticides and Beneficial Organisms". Entomophaga, v.39, p.7-119, 1994.

HENEBERRY, T.J.; JECH, L.F. Cotton aphid biology and honey-dew production. 2001. Disponível em: <http://ag. arizona.edu/pubs/crops/az1224/>. Acesso em: 30 nov. 2005.

ISIKBER, A.A.; COPLAND, M.J.W. Effects of various aphid foods on Cycloneda sanguinea. Entomologia Experimentalis et Applicata, v.102, n.1, p.93-97, 2002.

KABISSA, J.C.B.; YARRO, J.G.; KAYUMBO, H.Y.; JULIANO, S.A. Seasonal abundance of chrysopids (Neuroptera: Chrysopidae) preying Helicoverpa armigera (Hubner) (Lepidoptera: Noctuidae) and Aphis gossypii (Glover) (Homoptera: Aphididae) on cotton in eastern Tanzania. Crop Protection, v.15, n.1, p.5-8v 1996.

MAIA, V.B.; BUSOLI, A.C.; DELABIE, J.H.C. Seletividade fisiológica de Endossulfan e Deltametrina às operárias de $A$. chartifex spiriti For. (Hymenoptera, Formicidae) em Agroecossistema cacaueiro do Sudeste da Bahia. Neotropical Entomology, v.30, p.449-454, 2001.

MATTHEWS, G.A. Early season pests. In: MATTHEWS, G.A. (Ed.). Cotton insects pests and their management. Berkshire: Longman Scientific \& Technical, 1989. p.16-26.
OBRYCHI, J.J.; KRING, T.J. Predaceous coccinellidae in biological control. Annual Review of Entomology, v.43, p.295-321, 1998.

PENNA, J.C.V. Principais pragas da cultura e seu manejo. In: ARANTES, N. E. et al. Algodão e Soja. Belo Horizonte: Apesmg, 1998. p.34-37

RAMALHO, F.S.; JESUS, F.M.M.; GONZAGA, J.V. Táticas de manejo integrado de pragas em áreas infestadas pelo bicudo-do-algodoeiro. Pesquisa Agropecuária Brasileira, v.25, n.5, p.677-690. 1990.

SANTOS, W.J. Pragas do algodoeiro. In: Mato Grosso Liderança e Competitividade. Rondonópolis: Fundação MT/Embrapa, 1999. p.113-149. (Boletim, 3).

SCARPELLINI, J.R.; ZANETTI, L.F.; RATERO, W.D. Avaliação da seletividade de carbosulfan, acetamiprid, profenofos, zetacipermetrina e bifentrina no complexo de inimigos naturais de pragas (predadores) na cultura do algodoeiro. In: CONGRESSO BRASILEIRO DE ALGODÃO, 3., 2001, Campo Grande, MS. Anais. Campo Grande, 2001. p.123-126.

SCARPELLINI, J.R.; FARIA, A.M.; RODRIGUES, F.E. Seletividade fisiológica de aficidas sobre o complexo de inimigos naturais de pragas do algodoeiro utilizando-se diferentes métodos de amostragem. In: CONGRESSO BRASILEIRO DE ALGODÃO, 4., 2003, Goiânia, GO. Anais. Goiânia, 2003. 1 CD-Rom.

SCARPELLINI, J.R., ZANETTI, L.F.; CABRAL, S.B. Flonicamid 500 WG no controle de pulgões Aphis gossypii Glover (Hemíptera, Aphididae) e seletividade fisiológica sobre a joaninha Cycloneda sanguinea L. (Coleoptera, Coccinellidae) em algodoeiro. 2005. In: CONGRESSO BRASILEIRO DE ALGODÃO, 5., 2005, Salvador, BA. Anais. Salvador, 2005. 1 CD-Rom.

SOARES, J.J.; BUSOLI, A.C. Comparação de métodos de amostragem para artrópodes predadores associados ao algodoeiro. Anais da Sociedade Entomológica do Brasil, v.24, n.1, p.172-183, 1995.

SOARES, J.J.; BUSOLI, A.C. Efeito de inseticidas em insetos predadores em culturas de algodão. Pesquisa Agropecuária Brasileira, v.35, n.9, p.1889-1894, 2000.

SUIII, E.R.; BESERRA, V.A.; RIBEIRO, P.H. ; SILVASANTOS, P.V. DA; SILVA, K.F.A. DE; MACEDO, T.; PIRES, C.S.S.; SCHMIDT, F.G.V.; FONTES, E.M.G.; LAUMANN, R.A. Avaliação do controle biológico natural do pulgão, Aphis gossypii Glover (Homoptera: Aphididae) $e$ lagarta do curuquerê, Alabama argillacea Hübner (Lepidoptera. Noctuidae) na cultura do algodoeiro. Brasilia: Embrapa Recursos Genéticos e Biotecnologia, 2005. (Boletim de Pesquisa e Desenvolvimento n.113). 
VERGARA RUIZ, R.; GALEANO, P.E.O. Interacciones poblacionales entre áfidos y sus enemigos naturales en algodonero, en dos zonas del Tolima. Revista Colombiana de Entomologia, v.20, n.1, p.15-22, 1994.

WEATHERSBEE III, A.A.; HARDEE, D.D. Abundance of cotton aphids (Homoptera: aphididae) and associated biological control agents on six cotton cultivars. Journal of Economic Entomololyy v.87, n.1, p.258-65, 1994.

Recebido em 2/5/06

Aceito em 15/4/08 